\title{
ASSISTÊNCIA À CRIANÇA HOSPITALIZADA: REFLEXÕES ACERCA DA PARTICIPAÇÃo DOS PAIS*
}

\author{
Regina Aparecida Garcia de Lima** \\ Semiramis Melani Melo Rocha** \\ Carmen Gracinda Silvan Scochi**
}

\begin{abstract}
LIMA, R.A.G.; ROCHA, S.M.M.; SCOCHI, C.G.S. Assistência à criança hospitalizada: reflexões acerca da participação dos pais. Rev.latino-am.enfermagem, Ribeirão Preto, v. 7, n. 2, p. 33-39, abril 1999.
\end{abstract}

A assistência à criança hospitalizada vem sofrendo transformações significativas e, numa perspectiva mais atual, a estratégia central parece incentivar os pais ou responsáveis a permanecerem com suas crianças durante as internações. O objetivo desta investigação é analisar a participação dos pais no processo de hospitalização de seus filhos. Optamos pela metodologia qualitativa, tendo como referencial teórico-metodológico o Modelo de Organização Tecnológica do Trabalho. A partir da conformação do processo de trabalho assinalamos que trazer a mãe para a enfermaria não é uma questão simples pois implica na reorganização do trabalho em nível teórico e prático. Conceitos como cooperação e parceria estão sendo construídos, entretanto, compartilhar saberes, poderes e espaços, também não se constitui ato simples, decorrente da adesão a um discurso. Implica, sim, em mudanças de valores e atitudes, tanto por parte dos pais como dos profissionais.

UNITERMOS: criança hospitalizada, participação dos pais, enfermagem pediátrica

\section{INTRODUÇÃO}

A assistência à criança hospitalizada, nos países ocidentais, vem sofrendo transformações significativas, principalmente, a partir de fins do século XIX. Estas mudanças estão diretamente relacionadas ao modo de produção, ao desenvolvimento da prática médica e ao valor e significado que a sociedade dá à criança.

A literatura norte-americana, descreve que até 1930 a assistência de enfermagem à criança hospitalizada, tinha por finalidade prevenir a transmissão de infecção através do isolamento rigoroso. Esta forma de assistir afastou a mãe e familiares de um envolvimento com a criança e com os profissionais.

Nesta perspectiva, a importância do crescimento e desenvolvimento, das reações psicológicas infantis, da necessidade de educar e o impacto emocional da doença e da hospitalização na criança e nos pais eram pouco considerados. O legado desta forma de assistência, que afasta a mãe e familiares de um envolvimento com a criança e profissionais, ainda tem vestígios em nossos dias.

A partir de 1940, nos Estados Unidos, ocorrem mudanças de atitudes em relação à assistência à criança hospitalizada. Esta, torna-se mais complexa, acompanhando os avanços da prática médica, que se desenvolve para dar respostas às necessidades sociais que emergem, principalmente, com a Segunda Guerra Mundial. A enfermeira utiliza este novo conhecimento que advém da evolução dos conceitos da Fisiologia e Patologia, que por sua vez encaminham para aperfeiçoamentos de diagnóstico médico, tratamento e profilaxia das doenças. O hospital amplia o seu nível de complexidade.

Segundo DARBYSHIRE (1993, 1994), o declínio das doenças infecciosas, a introdução do antibiótico e tecnologias inovadoras contribuíram para uma revisão do afastamento dos pais e familiares durante a hospitalização de seus filhos. No entanto, a transformação no conceito de criança, agora vista como um ser em crescimento e desenvolvimento, não só com necessidades biológicas, mas também psicológicas, sociais e emocionais, foi o mais efetivo catalizador para esta mudança.

A preocupação com o crescimento e desenvolvimento na assistência à criança hospitalizada foi impulsionada pelo relatório publicado, em 1951, pela Organização Mundial de Saúde sobre a privação materna como fator etiológico perturbador da saúde mental.

\footnotetext{
* Trabalho subvencionado pelo CNPq (Processo nº 524507/96-1)

** Docentes da Disciplina Enfermagem Pediátrica e Neonatal da Escola de Enfermagem de Ribeirão Preto da Universidade de São Paulo e Pesquisadoras do Grupo de Estudos em Saúde da Criança e do Adolescente
} 
Outro marco na organização das práticas de assistência à criança hospitalizada foi a publicação do Relatório Platt (MINISTRY OF HEALTH, 1959), em 1959, na Inglaterra. Este documento trouxe à tona a preocupação com o bem-estar da criança internada em instituições hospitalares e levou pais e profissionais a discutirem e analisarem o processo de hospitalização, procurando alternativas para "humanizar" esta experiência.

Uma variedade de estratégias tem sido apontadas, principalmente na Inglaterra, mas também em outros países, entre os quais se inclui o Brasil. A estratégia central parece incentivar e encorajar os pais ou responsáveis a permanecerem com suas crianças durante as hospitalizações e, mais recentemente, a participarem dos cuidados dispensados a elas (DARBYSHIRE, 1994). No entanto, na atualidade, a prática assistencial brasileira na maioria das instituições não incorporou, sistematicamente, a presença da mãe ou responsável no cuidado. A medicina clínica, através do diagnóstico e terapêutica, responde prioritariamente às necessidades do corpo biológico e pouco valoriza, neste processo, as outras necessidades de um ser em fase de crescimento e desenvolvimento, que também quer brincar, ter autonomia, estabelecer vínculos, ser respeitado, receber e dar afeto, mesmo quando doente.

\section{O contexto brasileiro}

Diversos autores (BIERMANN, 1980; SCHMITZ, 1989; CYPRIANO \& FISBERG, 1990; LIMA, 1995 e 1996; COYNE, 1995), são unânimes em considerar que a separação da mãe é o fator que provoca maiores efeitos adversos no processo de hospitalização da criança, principalmente naquelas menores de seis anos de idade.

A adoção de um sistema de alojamento conjunto pediátrico (termo usado como sinônimo de mãe acompanhante, internação conjunta mãe-filho e mãe participante) em que a mãe ou responsável pode acompanhar a criança durante os episódios de hospitalização, é uma estratégia que possibilita a redução do estresse emocional, tanto da criança como da família, reduz a incidência de infecção cruzada e diminui o tempo de internação, favorecendo conseqüentemente a rotatividade e disponibilidade de leitos infantis (SÃO PAULO, 1989).

No Estado de São Paulo, é direito da mãe ou outro familiar, acompanhar sua criança durante a internação, situação esta oficializada em 12 de outubro de 1988 (SÃO PAULO, 1989). Entretanto, a implantação desta modalidade de assistência ainda não é realidade em várias instituições deste e de outros estados brasileiros, mesmo reconhecendo-se que a internação conjunta mãe-filho pode ser o método mais efetivo para reduzir os traumas psicológicos da hospitalização.

A Constituição do Brasil de 1988 incorpora como prioridade a proteção dos direitos da criança e do adolescente e o atendimento de suas necessidades básicas. Assim, em 13 de julho de 1990 foi promulgada a lei ${ }^{\circ}$ 8069 que regulamenta o Estatuto da Criança e do Adolescente e dispõe, no seu Artigo 12, que (...) "os estabelecimentos de saúde devem proporcionar condições para a permanência, em tempo integral, de um dos pais ou responsável, nos casos de internação de crianças e adolescentes" (BRASIL, 1991, p.16).

Desta forma, pela legislação, ocorre a universalização dos direitos da criança e do adolescente de usufruírem do sistema de alojamento conjunto pediátrico, contando com o acompanhamento da mãe ou responsável durante os processos de hospitalização. Em geral, esta possibilidade ainda encontra-se no plano das idéias e não da ação.

\section{O ESTUDO}

A hospitalização é uma experiência estressante que envolve profunda adaptação da criança às várias mudanças que acontecem no seu dia-a-dia. Contudo, pode ser amenizada pelo fornecimento de certas condições como: presença de familiares, disponibilidade afetiva dos trabalhadores da saúde, informação, atividades recreacionais, entre outras. O objetivo desta investigação é analisar a participação dos pais no processo de hospitalização de seus filhos.

Este estudo é de natureza qualitativa e utilizamos como referencial teórico o Modelo de Organização Tecnológica do Trabalho, elaborado por GONÇALVES (1986), que se fundamenta na dialética marxista.

Salientamos que este trabalho é parte de um estudo mais amplo sobre assistência integral à criança hospitalizada que tem como objetivo específico identificar quais são as necessidades das crianças hospitalizadas, enquanto seres em crescimento e desenvolvimento.

\section{O local do estudo}

O trabalho foi realizado no Hospital das Clínicas da Faculdade de Medicina de Ribeirão Preto da Universidade de São Paulo - HCRP, situado na cidade de Ribeirão Preto, localizada a Nordeste do Estado de São Paulo, distante $320 \mathrm{Km}$ da capital, fundada em 1856. Sedia a VI Região Administrativa do Estado, formada por sessenta municípios e é polo de produção de canade-açúcar para industrialização, algodão herbáceo, soja, milho, café, suco de laranja, aparelhos eletrodomésticos 
e hospitalares. É importante centro educacional, médicoassistêncial e comercial (RIBEIRÃO PRETO, 1991).

O HCRP é um hospital de ensino, de referência terciária e sua área de abrangência vai além das fronteiras do Estado de São Paulo, recebendo inclusive pacientes de outros países da América Latina. Oferece assistência médica de emergência, ambulatorial e internação programada além de propiciar atividades de capacitação de recursos humanos e pesquisa. É um hospital público estatal, classificado como de porte especial*** e o de maior complexidade da região, com sofisticados recursos tecnológicos para diagnóstico e terapêutica. Atende a uma clientela heterogênea do ponto de vista sócio-econômico, prevalecendo o atendimento gratuito, com fins acadêmicos e de pesquisa. É campo de estágio para graduandos de Medicina, Enfermagem e Psicologia; para residentes de Medicina, Psicologia, Fisioterapia e estagiários dos cursos de Auxiliar e Técnico de Enfermagem. Em agosto de 1996, contava com 665 leitos sendo que 35 eram infantis, alocados especificamente na Clínica Pediátrica que tem o Programa Mãe Participante.

Nesta unidade são hospitalizadas, preferencialmente, crianças com patologias crônicas e mais complexas que necessitam de reinternação freqüente. É uma das poucas instituições da região de Ribeirão Preto onde internam crianças com câncer além de dispor de unidades especializadas e sofisticadas para atendimento de pacientes transplantados ou em hemodiálise.

Salientamos que esta unidade já nos era familiar pois é campo de ensino clínico da disciplina Enfermagem Pediátrica e Neonatal da qual fazemos parte. DARBYSHIRE (1994) discute a questão da realização do trabalho de campo em locais previamente conhecidos do pesquisador. Para o autor um aspecto benéfico nesta situação é principalmente a negociação para o acesso do pesquisador ao campo.

No caso da presente pesquisa, a entrada no campo foi facilitada, pois nossa presença é habitual e de certa forma já foi processada pelos trabalhadores. $\mathrm{Na}$ nossa opinião, a familiaridade com o local de coleta de dados também possibilitou que a relação entre pesquisador e sujeitos pesquisados fosse desenvolvida de forma menos tensa encaminhando-nos para a seleção da técnica de coleta de dados que foi a observação participante.

\section{Participantes}

Participaram do estudo dez crianças, acompanhadas de suas mães ou responsáveis, internadas na Clínica Pediátrica do HCRP. A seleção dos binômios mãe-filho foi intencional, tendo por base critérios previamente formulados em relação aos quais eles seriam os mais representativos.

Quanto ao perfil das crianças selecionadas, a idade variou de um a doze anos; sete eram do sexo feminino e três do masculino. Oito crianças já haviam passado por outras internações (casos crônicos) e duas vivenciavam a primeira, com mais de trinta dias de hospitalização. Apenas uma criança tinha como acompanhante a tia, as demais a mãe.

\section{Coleta de dados}

A coleta de dados, realizada através da observação participante, foi desenvolvida pela própria pesquisadora e uma auxiliar de pesquisa, também enfermeira, com experiência profissional em Enfermagem Pediátrica.

O período de coleta de dados estendeu-se de 09.01 a 12.04.95 e observamos uma criança de cada vez, por cinco dias consecutivos, de segunda a sexta-feira, nos plantões da manhã, tarde e vespertino. O total de horas por período não foi fixado pois dependeria de cada situação. A observação foi centrada na criança (unidade de observação) e nas pessoas (mães e trabalhadores da saúde) que com ela se relacionavam. Adotamos um posicionamento móvel pois acompanhávamos e registrávamos seus movimentos, falas, ações, aparência física, expressões, espaço físico e de relações. As observações foram registradas nos diários de campo dos respectivos observadores.

O primeiro passo para operacionalizar esta fase foi o encaminhamento do projeto à Comissão de Normas Éticas do HCRP obtendo, posteriormente, permissão oficial para a realização do estudo.

Antes de iniciarmos o período de observação, apresentávamo-nos às mães, explicávamos nossos propósitos de pesquisa e solicitávamos seu consentimento para a realização da investigação, todas concordaram.

$\mathrm{Na}$ pesquisa qualitativa, como em outras abordagens, o respeito ao anonimato e sigilo são particularmente importantes. Em pequenos espaços sociais, em geral, através de alguns descritores é possível identificar os sujeitos. Algumas precauções foram adotadas afim de proteger a identidade dos participantes. Assim, a identificação das crianças foi feita através de letras, seguindo a seqüência alfabética, não havendo correspondência entre as letras e o nome; as observações referentes à elas foram designadas por números. Com

\footnotetext{
*** Segundo a publicação do Ministério da Saúde sobre terminologia básica em saúde, hospital de porte especial é aquele com capacidade instalada acima de 500 leitos (BRASIL, 1985)
} 
este procedimento procurou-se evitar que as identidades pessoais fossem associadas às informações por elas fornecidas.

\section{Análise}

A técnica de observação participante produz um grande volume de informações descritivas e narrativas. Iniciamos esta etapa paginando os diários de campo numa seqüência temporal. Em seguida, digitamos o conjunto das observações para procedermos a ordenação dos dados coletados a partir de agrupamentos, neste primeiro momento, retirados do conjunto das observações. Construímos assim, diversos títulos para aglutinar os dados brutos, elaborando-se uma primeira visão do conjunto.

A realização da leitura flutuante deste conjunto de títulos associada à identificação de aspectos relevantes para a análise, conduzidas pelo referencial teórico adotado e pelo objetivo colocado para o desenvolvimento deste estudo, possibilitou-nos apreender as estruturas de relevância, que segundo MINAYO (1989) caracterizamse pela incidência de ênfase em determinados aspectos da realidade, indicando a base de confronto do material empírico com as teorias existentes sobre o assunto.

Uma das estruturas de relevância foi a participação dos pais, tema desta pesquisa, lembrando que este trabalho é parte de um estudo mais amplo sobre assistência integral à criança hospitalizada, conforme comentado anteriormente.

\section{RESULTADOS E DISCUSSÃO}

A Clínica Pediátrica localiza-se no $7^{\circ}$ andar do HCRP, dividindo este espaço com o Berçário de AltoRisco. Conta com trinta e cinco leitos nos quais são internadas crianças e adolescentes com patologias crônicas e mais complexas que necessitam de reinternações freqüentes. É uma das poucas unidades da região que presta assistência à criança com câncer sendo referência também para pacientes com fibrose cística (mucoviscidose). Este serviço mantém o Programa MãeParticipante, ou seja, é permitido que a mãe ou responsável acompanhe sua criança ou adolescente durante o processo de hospitalização.

A Clínica Pediátrica não foi planejada para atender crianças e adolescentes apresentando inadequação de planta física. Além disso, não comporta as necessidades do Programa Mãe Participante (SÃO PAULO, 1989) que prevê planta física adequada para acomodação das mães (por exemplo, sala de repouso e sanitários privativos).
No espaço hospitalar encontram-se diferentes tipos de trabalhadores que se ocupam de funções determinadas sob a égide de normas autoritárias e entre os quais se estabelecem relações de poder dadas pela divisão social e técnica do trabalho.

A composição dos profissionais envolvidos na assistência hospitalar varia de acordo com a produção de serviços própria de cada instituição. Assim, na unidade pediátrica analisada, o médico, a psicóloga, a nutricionista, a assistente social e a enfermagem, com suas diversificações em categorias, são os agentes envolvidos no processo de trabalho, cabendo a cada um desenvolver determinadas atividades de acordo com a capacitação profissional. Somam-se a estes, trabalhadores ligados ao setor de hotelaria, serviço de lavanderia, zeladoria e o pessoal administrativo.

Por esta diversidade de profissionais, o modelo de produção de cuidados orienta-se, principalmente, para o desenvolvimento de cuidados especializados e de maior complexidade. A lógica social que o organiza mantém como princípio dominante da divisão do trabalho a oposição entre comando (trabalho intelectual) e execução (trabalho manual). Na decomposição da assistência hospitalar global em atividades diversificadas e isoladas reserva-se ao ato médico, propriamente dito, posição nuclear pois a elaboração do diagnóstico e a escolha da terapêutica são resultados de um trabalho intelectual de síntese.

Consonante com esta situação verificamos que a divisão de tarefas está presente na assistência. Assim, o processo de trabalho é fragmentado pelas diversas intervenções terapêuticas do médico, da enfermagem, da nutricionista, da psicóloga... Em geral estes profissionais não tomam conhecimento do processo por inteiro. Cada um atua de acordo com um saber específico, de forma independente, talvez apenas complementar, no sentido de somatória de ações.

$\mathrm{Na}$ atualidade, constatamos que a mãe é outro agente do trabalho, pois the é delegada uma série de atividades que anteriormente eram de responsabilidade da enfermagem. GONÇALVES (1986), na discussão sobre divisão técnica e social do trabalhado e, mais especificamente, trabalho "manual" e "intelectual", ao analisar o trabalho da enfermeira, aponta que as funções relativamente "mais manuais" associadas ao processo terapêutico e de infra-estrutura vão se transferindo para a enfermeira que, por sua vez, detém desta parte, as funções "mais intelectuais" (administração e gerenciamento) e delega a seus auxiliares as tarefas mais parcelares e "manuais" deste mesmo trabalho.

Parece que esta divisão está presente no microespaço de relações da enfermagem/mãe, pois também foi delegado às últimas, atividades mais simples (cuidados higiênicos) e alimentação do processo de trabalho, 
embora pela complexidade tecnológica da assistência hospitalar, característica das instituições de nível terciário, a alimentação, por vezes, é administrada através de sondas ou o banho é realizado com outra técnica, que não a cotidiana, em função dos monitores, catéteres ou soroterapia.

A. aceitou apenas $40 \mathrm{ml}$ do leite por via oral, mãe coloca o restante no frasco de alimentação por sonda, conecta o equipo, faz o nível, retira o ar e desclampa a sonda nasogástrica instalando o leite; controla o gotejamento sem auxílio de relógio. (...) após terminar, retira o frasco e introduz $10 \mathrm{ml}$ de água fervida para "lavar" a sonda (Obs. 1, p.60).

São $8 \mathrm{~h} 30 \mathrm{~min}, \mathrm{~F}$. está no décimo dia da internação, tem 4 anos e 3 meses e diagnóstico de câncer com metástases. Apresenta-se choroso, queixando de dor abdominal, com sonda nasogástrica e soroterapia em membro inferior direito. Mãe prepara material para o banho e na seqüência inicia o procedimento demonstrando cuidado e atenção com o soro e a sonda. Criança continua reclamando de dor, principalmente quando é mudada de posição. Mãe solicita ajuda da auxiliar de enfermagem apenas para trocar os lençóis (Obs. 6, p.237).

Além destas atividades, tem sido delegada à mãe outras como: administração de medicação por via oral, banhos terapêuticos, aerosol, tapotagem. Nas situações de medicações por via oral, a auxiliar de enfermagem prepara-as e a mãe administra-as. B. está no leito, em decúbito ventral e mãe realiza tapotagem. Ambas estão calmas; mãe orienta criança a tossir e expectorar, $B$. atende prontamente e elimina grande quantidade de secreção espessa esverdeada. Em seguida, auxiliar de enfermagem, escalada para medicação, entrega à mãe medicação para ser dada por via oral, B. aceita após insistência (Obs. 2, p.106).

Técnica de enfermagem chega a enfermaria com o carrinho de medicação, inicia por $F$., dilui o comprimido em água, coloca açúcar e aspira a solução em uma seringa. Aproxima-se da mãe e pede que ofereça sem forçar; olha para a criança e diz (...) "o remédio está gostoso pois está docinho, experimente prá você ver". (...) após instalar antibiótico (no microgotas) por via endovenosa, orienta a mãe a controlar o gotejamento do soro e "lavar" a veia no final (...). Ao término da medicação, mãe procede como foi orientada, abre a pinça do soro glicosado para que $10 \mathrm{ml}$ penetre no microgotas, aumenta 0 gotejamento e sai para chamar a funcionária da enfermagem (Obs. 6, p.267).

Mãe de A. é responsável pelo banho de permanganato de potássio, neste momento não foi orientada mas faz o cuidado corretamente. Coloca A. no berço, retira sua camiseta, envolve o pescoço com uma fralda embebida na solução; (...) quando muda de cor, inicia novamente o procedimento. Criança calma e colaborativa (Obs. 1, p.54).

O "treinamento" da mãe é feito, na maioria das vezes, pela atendente ou auxiliar de enfermagem, no entanto, a supervisão do cuidado raramente é realizada. A partir do momento que a enfermeira delegou à mãe a execução de uma técnica ou cuidado, é sua a responsabilidade de desenvolvê-los. A prática da supervisão, da orientação e da educação continuada são instrumentos de trabalho da enfermeira para a capacitação de recursos humanos e, neste momento de transformação do processo de trabalho, no qual a mãe está sendo inserida, é fundamental que ela ocupe-se sistematicamente da orientação e supervisão destas mães pois, teoricamente, é o profissional melhor preparado.

A partir do momento que a enfermagem delega à mãe determinadas ações, ela (enfermagem) estará mais sujeita ao controle, o qual não é sempre bem aceito ou compreendido. Quando a enfermagem delega cuidados, ela também fornece à mãe instrumentos que a levarão a controlar o trabalho da enfermagem mais tarde, como na seguinte situação: $\mathbf{C}$. tem plasma correndo em veia puncionada no membro superior direito; está no leito, em posição semi-sentada, folheando uma revista, Auxiliar de enfermagem escalada para medicação, entra na enfermaria às $16 \mathrm{~h} 55 \mathrm{~min}$ e a mãe comenta "hoje você está adiantada, o plasma foi instalado às $15 \mathrm{~h}$ (a prescrição era de uma ampola de diurético na metade do volume do plasma). A funcionária da enfermagem responde asperamente (...) "não mãe! $O$ plasma foi instalado às $14 \mathrm{~h} 55 \mathrm{~min}$ para correr em quatro horas e agora são exatamente 16h55min" (Obs. 3, p.65).

Parece-nos que a enfermeira ao delegar atividades indiscriminadamente para seus auxiliares e, neste momento, também para a mãe, pode deparar-se com situações que se opõem à lei do exercício profissional. Exemplificando, é possível chegar-se ao extremo de delegar à mãe cuidados de responsabilidade dos profissionais. Acreditamos que estudos empíricos devam ser realizados objetivando uma melhor compreensão desta problemática, presente no processo de trabalho da enfermeira pediátrica, mas não exclusiva dele.

COYNE (1995) fez uma ampla revisão da literatura demonstrando que a participação dos pais é uma questão complexa que tem sido tratada de forma fragmentada não reconhecendo a natureza da relação entre pais e enfermeiras como uma parceria integrada. $\mathrm{O}$ conhecimento acerca da atitude da enfermagem nesta relação é limitado, faltando indicadores claros de como a participação dos pais pode ser facilitada e apoiada num ambiente institucional.

Além dos cuidados relacionados ao corpo biológico, identificamos a participação das mães nas atividades recreacionais de seus filhos. Este envolvimento 
possibilita o estreitamento dos laços entre ambos mas também é fonte de lazer para a mãe já que ela tem poucas oportunidades de diversão na enfermaria. A atividade recreacional é parte importante do processo terapêutico da criança hospitalizada mas é, também, parte essencial na construção, mais especificamente, na reconstrução de sua identidade. Na perspectiva das mães, a atividade recreacional é uma estratégia para distrair os filhos e minimizar o estresse presente nos processos de hospitalização. Utilizam-se dela para reaproximarem-se do cotidiano e segundo DARBYSHIRE (1994), as mães têm uma percepção clara de que o brinquedo não é meramente uma atividade para passar o tempo mas é um importante estímulo para o desenvolvimento intelectual, afetivo, emocional e social de suas crianças.

Desta forma, durante a hospitalização do filho, os pais procuram formas de atenuar suas necessidades físicas, centradas na terapêutica mas também estão atentos ao seu bem-estar emocional. Eles parecem conseguir integrar na assistência, aspectos emocionais, psicológicos e do corpo biológico propriamente dito. Esta constatação tem surpreendido os agentes de saúde pois, em geral, eles consideram os pais como receptáculo de informações e ordens, com pouca capacidade de compreender e agir durante os processos de hospitalização de seus filhos.

Segundo CALLERY \& SMITH (1991), a participação dos pais no processo de hospitalização de seus filhos é amplamente defendida pelos profissionais de saúde, no entanto, não está delimitada a extensão desta participação. Nesta perspectiva, os autores sugerem que a negociação de papéis poderia reduzir o potencial para o conflito e prevenir problemas presentes na prática.

\section{CONSIDERAÇÕES GERAIS}

Tradicionalmente, a assistência à criança hospitalizada não incluía a permanência dos pais e as propostas mais recentes de fazê-los participantes implica em um novo modo de pensar e organizar o trabalho. Nesta perspectiva, a visão de cooperação e parceria está sendo construída e como tal ainda não é consenso ou mesmo aceita em muitas instituições.
Quando a assistência hospitalar está centrada nas necessidades da criança doente e não apenas na doença, quando é permitido aos pais participarem do cuidado, eles sentem-se mais tranqüilos e confiantes. A organização do processo de trabalho, geralmente, parece indicar a intenção de monopólio do cuidado pelos profissionais, no entanto, para uma assistência mais integral, parece-nos que a incorporação dos pais no processo é importante.

Trazer a mãe ou responsável para a enfermaria não é uma questão simples pois implica na reorganização do processo de trabalho em nível teórico e prático. Um fato a se considerar é que a permanência dos pais está imprimindo uma outra dinâmica no processo pois eles não estão desenvolvendo apenas habilidades técnicas, mas estão também "conhecendo" o cotidiano do hospital e da terapêutica e, lentamente, reivindicam participação na assistência para além dos cuidados básicos como alimentação e higiene.

Compartilhar saberes, poderes e espaços, não é um ato linear e simples, decorrente da adesão a um discurso. Implica, sim, em mudanças de valores e atitudes por parte dos pais e profissionais. No entanto, o conjunto de explicações encontrado na literatura nacional é, ainda, insuficiente para a compreensão deste fenômeno tão complexo. Para ampliar esta discussão sugerimos que se promovam pesquisas sobre relações de poder tornando transparente o papel de cada participante no espaço hospitalar.

Finalizando, argumentamos que a assistência hospitalar, cuja dinâmica confere ao cuidado especificidades singulares, seja pautada pela integração de saberes e ações. Nesta perspectiva o que se requer é um corpo de conhecimentos mais amplo do que o utilizado atualmente. Assim, além do clássico biológico, incluindo a Fisiologia, Patologia e a Clínica, sejam incorporados outros saberes que desvendem as relações interpessoais permitindo avançar na postulação de um quadro teórico mais abrangente. Esta possibilidade poderá contribuir para alterar a visão corrente que atribui à participação e envolvimento dos pais um valor essencialmente instrumental e funcional.

\section{CARE TO HOSPITALIZED CHILDREN: REFLECTIONS ABOUT PARENTS' PARTICIPATION}

The care of a child admitted in the hospital is going through meaningful changes and, in a more actual approach, the main strategy seems to stimulate parents or relatives to stay with their children in the hospital. The purpose of this study is to analyse parents' participation in the process of staying in the hospital with their children. We have chosen the qualitative method and used as a theoretic - methodological reference the Model of Technological Work Organization. Based on the process of work, we have pointed out that bringing mothers to the ward is not so simple as it interferes in the reorganization of the work at the theoretical and practical levels. Concepts such as co-operation and partnership are built, however, sharing knowledge, power and room is not a simple action. It implies in changes of values and postures by parents and professionals. 


\section{ASISTENCIA AL NIÑO HOSPITALIZADO: REFLEXIONES A CERCA DE LA PARTICIPACIÓN DE LOS PADRES}

La asistencia al niño hospitalizado ha sufrido transformaciones significativas y en una perspectiva mas actual, a estrategia central parece incentivar a los padres o personas responsables a que permanezcan con sus niños durante las hospitalizaciones. El objetivo de esta investigación es evaluar la participación de los padres en el proceso de hospitalización de sus hijos. Optamos por la metodologia cualitativa, teniendo como referencial teórico - metodológico el Modelo de Organización Tecnológica del Trabajo. A partir de la conformación del proceso de trabajo encontramos que traer a la madre para la habitación no es una cuestión simple, pues implica una reorganización del trabajo a nível teórico y práctico. Conceptos como cooperación y compañia están siendo construidos, sin embargo, compartir saberes, poderes y espacios no constituye un acto simple, en consecuencia de la adhesión a un discurso. Implica, si, un cambio de valores y actitudes, tanto de los padres como de los profesionales.

TÉRMINOS CLAVES: niño hospitalizado, participación de los padres, enfermería pediatrica

\section{REFERÊNCIAS BIBLIOGRÁFICAS}

01. BIERMANN, G. A criança e a hospitalização. Rev. Geográfica Universal, n. 3, p.54-60, 1980. I Documento Roche/.

02. BRASIL. Ministério da Saúde. Terminologia básica em saúde. 2.ed. Brasília: Centro de Documentação do Ministério da Saúde, 1985. 49p.

03. BRASIL. Ministério da Saúde. Estatuto da criança e do adolescente. Brasília: Ministério da Saúde, 1991. 110p.

04. CALLERY, P.; SMITH, L. A study of role negotiation between nurses and the parents of hospitalized children. J.Adv. Nurs., v.16, n.4, p.772-81, 1991.

05. COYNE, I. T. Parental partipacion in care: a critical review of the literature. J. Adv. Nurs., v.21, n.4, p. 716-22, 1995.

06. CYPRIANO, M. S.; FISBERG, M. Mãe Participante: benefícios e barreiras. J. Pediatr., São Paulo, v.66, n.4/5, p.92-7, 1990.

07. DARBYSHIRE, P. Parents, nurses and paediatric nursing: a critical review, J. Adv. Nurs., v.18, n.5, p.1670-80, 1993.

08. DARBYSHIRE, P. Living with a sick child in hospital: the experiences of parents and nurses. London: Chapman \& Hall, 1994. 225p.

09. GONÇALVES, R. B. M. Tecnologia e organização das práticas de saúde: características tecnológicas do processo de trabalho na rede estadual de centros de saúde de São Paulo. São Paulo, 1986. 399p. Tese (Doutorado). Faculdade de Medicina, Universidade de São Paulo.
10. LIMA, R.A.G. A enfermagem na assistência à criança com câncer. Goiânia: AB, 1995. 109p.

11. LIMA, R.A.G. Criança hospitalizada: a construção da assistência integral. Ribeirão Preto, 1996. 258p. Tese (Doutorado) - Escola de Enfermagem de Ribeirão Preto, Universidade de São Paulo.

12. MINISTRY OF HEALTH. Report on the welfare of children in hospital. London: HMSO, 1959.

13. MINAYO, M.C.S. O desafio do conhecimento: metodologia de pesquisa social (Qualitativa em saúde). Rio de Janeiro, 1989. 366p. Tese (Doutorado) - Escola Nacional de Saúde Pública, FIOCRUZ.

14. RIBEIRAO PRETO. Secretaria Municipal de Saúde. Plano diretor de saúde do município de Ribeirão Preto. Ribeirão Preto: Prefeitura Municipal de Ribeirão Preto, 1991. v.1. / Datilografado/

15. SÃO PAULO. (Estado). Leis, Decretos, etc. Resolução SS-165, 12 out. 1988. Diário Oficial do Estado, São Paulo, 14 mar. 1989. Seção 1, p.99.

16. SCHIMTZ, E.M.R. A problemática da hospitalização infantil: aspectos psicológicos. In: SCHIMTZ, E.M.R. et al. A enfermagem em pediatria e puericultura. São Paulo: Atheneu, 1989. 